Revista Calidad en la Educación Superior

Programa de Autoevaluación Académica

Universidad Estatal a Distancia, Costa Rica

ISSN 1659-4703

revistacalidad@uned.ac.cr

\title{
UNA VISIÓN SOBRE LA CALIDAD DE LA ATENCIÓN A LAS PERSONAS CON DISCAPACIDAD EN LA UNED
}

\author{
A INSIGHT ON THE QUALITY OF ATTENTION FOR PEOPLE WITH \\ DISABILITIES AT UNED
}

Adrián Solano Castro', asolano@uned.ac.cr Jorge Zeledón Solano ${ }^{2}$, jzeledon@uned.ac.cr Universidad Estatal a Distancia, Costa Rica

\author{
Volumen 6, número 1 \\ Mayo 2015 \\ Pp. $269-288$
}

Recibido: 15 de abril, 2015

Aprobado: 30 de abril, 2015

1 Adrián Solano Castro. Magister en Administración Educativa de la Universidad Estatal a Distancia. Actualmente, se desempeña como docente coordinador de la Cátedra de Administración Educativa de la Universidad Estatal a Distancia, Costa Rica.

2 Jorge Zeledón Solano. Magister en Educación a Distancia de la Universidad Estatal a Distancia. Actualmente, se desempeña como docente coordinador de la Cátedra de Filosofía de la Universidad Estatal a Distancia, Costa Rica. 


\section{Resumen}

Este artículo trata sobre la capacitación del personal docente de la Universidad Estatal a Distancia de Costa Rica en la atención de personas con discapacidad. Esta investigación se basa en una encuesta de carácter descriptivo y su objetivo es diagnosticar la actitud y la experiencia de los profesores y las profesoras de la UNED con relación a la población estudiantil que presenta alguna discapacidad. Se concluye que para lograr una atención de calidad se debe incrementar la especialización de los profesores y las profesoras en la atención a estudiantes con discapacidad.

Palabras claves: gestión, calidad en educación, personas con discapacidad, capacitación, educación a distancia

\section{Abstract}

The present article deals with training to professors at Distance State University of Costa Rica in the topic of adequate attention to people with special needs. A descriptive research was carried out. The objective of the research was to diagnose UNED professors' attitude as well as experience related to students with any kind of impairment. It was concluded that in order to give quality attention, it is necessary to increase the expertise of professors when it comes to deal with students with disabilities.

Keywords: Management, Quality of education, people with disabilities, training, distance education

En las últimas décadas la gestión universitaria ha tratado de aproximarse con más fuerza a temas relacionados con la gestión de la calidad, generando dinámicas para tratar de explicar y comprender cómo las instituciones construyen su modelo de gestión de la calidad. Sin embargo, estos esfuerzos se tornan más complejos por las profundas transformaciones en la sociedad contemporánea, a partir de lo cual también han cambiado los contextos de educación superior.

Sobre este fenómeno, en la Conferencia Mundial sobre la Educación Superior (UNESCO, 2009) se ofreció una visión del concepto de calidad en la educación superior, al respecto se indicó que 
La educación superior debe no sólo proporcionar competencias sólidas para el mundo de hoy y mañana, sino contribuir además a la formación de ciudadanos dotados de principios éticos, comprometidos con la construcción de la paz, la defensa de los derechos humanos y los valores en democracia...

En los últimos diez años se han realizado ingentes esfuerzos a fin de mejorar el acceso y garantizar la calidad. Este empeño debe continuar. Pero el acceso, por sí sólo, no es suficiente. Sera preciso hacer mucho más. Hay que llevar a cabo esfuerzos para lograr que los educandos obtengan buenos resultados (pp.2-3).

No obstante, si el modo de organizar las universidades permanece rígido y no se modifican las políticas de calidad y las prácticas educativas de atención a la diversidad, con su consecuente asignación de recursos, no se producirán avances que destierren la inequidad.

Este trabajo pretende ofrecer criterios para analizar la calidad y los alcances de la atención a personas con discapacidad. La motivación básica es reunir información que permita conocer qué estrategias en el área, tanto de orden administrativo como académico, se realizan para mejorar el abordaje inclusivo de los y las estudiantes con discapacidad.

\section{Referente teórico}

En general, en el ámbito de la educación superior el tema de la calidad es percibido como algo complejo por las múltiples aproximaciones al mismo. Por lo cual se ha desarrollado un amplio debate sobre la calidad en las universidades

De tal manera que entre las diferentes perspectivas y aproximaciones del concepto de la calidad en educación hay un consenso en que es un valor que se define considerando situaciones educativas específicas, y no debe entenderse como un valor absoluto. Los significados que se le atribuyen a la calidad 
dependerán de la perspectiva social y teórica desde la cual se hacen, de los sujetos que la enuncian (profesores y profesoras, padres y madres de familia, estudiantes, sectores productivos, Estado) y desde el lugar en el que se realiza.

Desde una perspectiva de evaluación de la calidad, para este trabajo se asume la calidad como una adecuación de propósitos. Esta definición asume un enfoque práctico sobre calidad, la refiere a los fines educativos señalados en el marco legal o a los fines que se propone la institución educativa (Juran, 1996; Cano, 1999; Giraldo, Abad y Díaz, 2005).

Considerar la discapacidad como tema de derechos humanos es una tendencia que surge en el ámbito internacional impulsado por los movimientos de personas con discapacidad desde la década de los años ochenta del siglo XX. Este nuevo abordaje ha significado un cambio de paradigma que comprende la filosofía de la igualdad de oportunidades como derecho humano.

En el articulado de la Convención Internacional de los Derechos Humanos para personas con discapacidad se estipulan los siguientes derechos: Derecho a la vida, derecho a la libertad, derecho a la libertad de tránsito, derecho a la libertad de expresión, integridad personal, derecho a no ser torturado ni sometido a tratos crueles y degradantes, derecho a vivir libre de violencia, derecho a la autonomía personal, derecho a la privacidad, derecho a la familia, derechos sexuales y reproductivos, derecho a la justicia, derechos políticos, derecho a la educación, derecho a la salud, derechos al trabajo. (ONU, 2007)

En Costa Rica la visión y conceptualización de la discapacidad desde el enfoque de los derechos humanos busca la inserción en todas las áreas, tales como educación, servicios de salud, empleo y transporte, para promover un desarrollo integral dentro de la sociedad de la población con discapacidad. 
Por lo tanto, en nuestro país, además de la Constitución Política, se cuenta con la Ley de Igualdad de Oportunidades para las Personas con Discapacidad, N. ${ }^{\circ} 7600$; de ella se puede extraer con especial interés en la idea de calidad el siguiente artículo:

\section{Artículo 14.- Acceso}

El Estado garantizará el acceso oportuno a la educación a las personas, independientemente de su discapacidad, desde la estimulación temprana hasta la educación superior. Esta disposición incluye tanto la educación pública como la privada en todas las modalidades del Sistema Educativo Nacional (Asamblea Legislativa, 1996, p.4)

Además, con el surgimiento de la legislación sobre los derechos de las personas con discapacidad, la Universidad Estatal a Distancia (UNED) implementó normativas con la finalidad de lograr políticas de carácter inclusivo. Se pueden identificar documentos que expresan esa tendencia, como en el Plan académico 2008-2011 (2008).

En los ejes transversales del Plan académico se expresa lo siguiente: "Una llamada de atención, sobre la necesidad de convertir la acción educativa en una acción profunda y globalmente humanizadora» (UNED, 2008, p.31). También en el eje de diversidad cultural se indica: "Se integra la consideración de la Ley de Igualdad de Oportunidades para las personas con discapacidad» (p.8).

En este mismo sentido, el Plan académico hace referencia a los derechos humanos, entendidos como un valor vinculado a la dignidad del ser humano y a sus aspiraciones de seguridad, justicia e igualdad. La UNED destaca y defiende la diversidad del ser humano, asimismo postula como valor incuestionable la igualdad de oportunidades. 
Es importante subrayar que el derecho a la educación está reconocido en los diversos instrumentos legales que velan por la población con discapacidad (Instituto Interamericano de Derechos Humanos, 2007).

\section{Metodología}

Esta investigación se desarrolla en el marco del enfoque cuantitativo, pues se recolectaron datos o percepciones sobre diferentes aspectos de la atención a la persona con discapacidad por parte del personal académico de la UNED y se realiza un análisis y medición de los mismos.

Para desarrollar el estudio, de acuerdo con Hernández, Fernández y Baptista (2010), se trabaja bajo un enfoque de investigación por encuesta con un diseño no experimental descriptivo transversal, porque se utilizó el cuestionario como instrumento de recolección de la información.

La población seleccionada son todos los profesores-tutores y todas las profesorastutoras de la UNED que fueron nombrados en el primer cuatrimestre de 2011. Se seleccionó esta población porque en su ejercicio profesional son los responsables directos de la atención a los y las estudiantes con discapacidad.

La muestra se seleccionó por criterio de conveniencia, que, de acuerdo con Creswell (2003), es el procedimiento de muestreo cuantitativo en el cual se seleccionan las personas informantes, porque están dispuestas y disponibles para ser estudiadas. Dicha muestra quedó conformada por veintitrés profesores-tutores y profesoras-tutoras del Programa de Estudios Generales, con un total de $30.4 \%$ de hombres y $69.9 \%$ de mujeres.

Es importante destacar que, al ser una muestra tomada por criterio de conveniencia (no aleatoria), los resultados obtenidos reflejan la realidad del grupo estudiado; no se pueden inferir resultados a la población. Sin embargo, aunque no se pueden generalizar los resultados que se obtuvieron, la descripción de ellos 
podría ser un insumo valioso para conocer la calidad de la atención a las personas con discapacidad en la UNED.

Se diseñó un instrumento con veintiún ítems en escala tipo Likert de cinco categorías, organizado en tres segmentos: el primero, de seis ítems, exploró sobre información general; el segundo, de ocho ítems, indagó acerca de las percepciones docentes sobre aspectos actitudinales de los y las docentes en la atención de estudiantes con alguna discapacidad; y el tercero, de siete ítems, examinó creencias docentes respecto a la atención de estudiantes con alguna discapacidad.

En cuanto al tratamiento de la información, se trabajan estadísticas descriptivas mediante el programa Excel, además se diseñan tablas y cuadros según distribución por sexo, edad, dependencia académica, años de servicio, calidad del servicio ofrecido a los y las estudiantes, adecuación del entorno, conocimiento de la normativa institucional, inducciones recibidas, percepción sobre la coordinación, aspectos de orden psicológico, dificultad para desarrollar la tutoría en presencia de personas con discapacidad, conocimiento de la existencia de protocolos de atención a las personas con discapacidad.

\section{Resultados}

De la totalidad de personas entrevistadas se observa que la mayor cantidad corresponde a hombres, con un $69.6 \%$, mientras que las mujeres representan el $30.4 \%$, tal como lo muestra la figura 1 . Estas cifras guardan relación con la distribución según sexo de los tutores y las tutoras de los demás programas de la universidad. 
Figura 1. Distribución según sexo tutores encuestados (I cuatrimestre 2011)

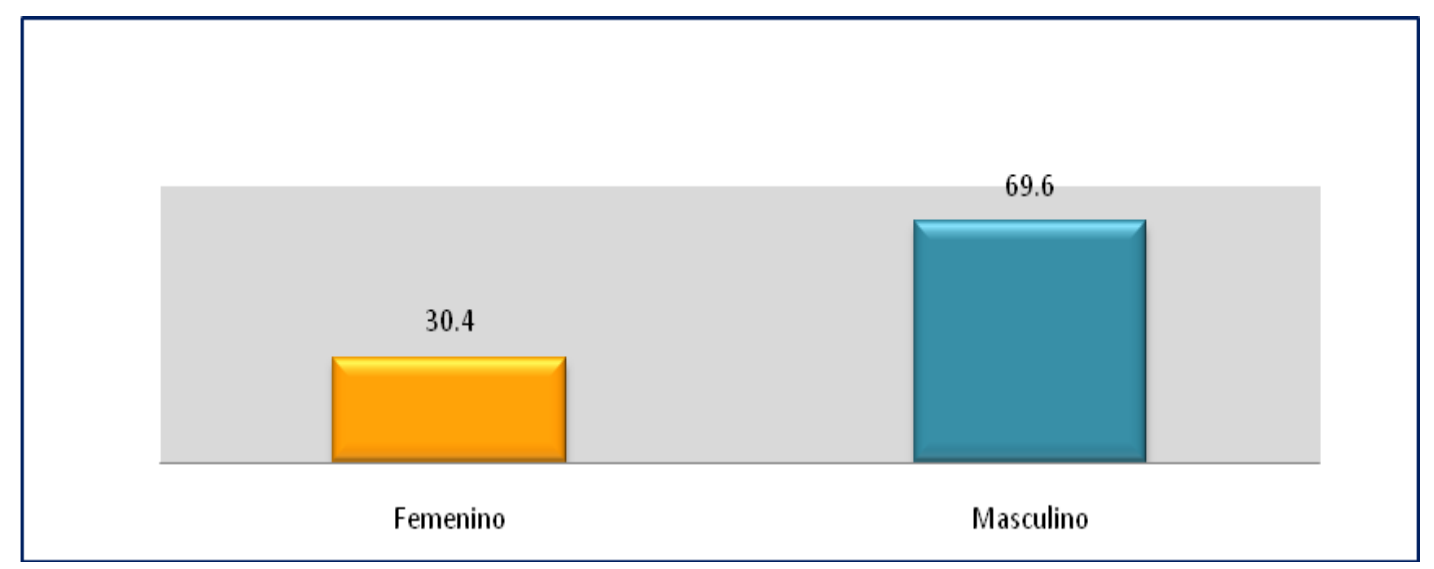

Fuente: Elaboración propia

El 30.4\% pertenece a la Cátedra de Filosofía, seguido de los tutores y las tutoras de las Cátedras de Historia y Métodos de Estudios a Distancia e Investigación con un $21.7 \%$ cada dependencia. Literatura con un $8.7 \%$ y un $17.5 \%$ no respondió.

\section{Tabla 1. Distribución por dependencia académica de tutores encuestados del Programa de Estudios Generales}

\begin{tabular}{l|lc}
\hline \multicolumn{1}{c|}{ Dependencia } & Absoluto & Relativo \% \\
\hline Cátedra Filosofía & 7 & 30.4 \\
Cátedra Historia & 5 & 21.7 \\
Cátedra Literatura & 2 & 8.7 \\
Cátedra de Métodos de Estudio a & 5 & 21.7 \\
Distancia e Investigación & & \\
No responde & 4 & 17.5 \\
\hline Total & 23 & 100.0
\end{tabular}

Fuente: Elaboración propia

Un $43.5 \%$ de las personas entrevistadas se encuentra en el rango de cero a cinco años de laborar en la UNED, mismo porcentaje que se encuentra en el rango de seis a diez años. Además, de la población entrevistada un $53 \%$ se encuentra entre 
los dos rangos de edad más elevados: $43-50$ años y 51 años o más, con un $26.1 \%$ para cada rango. Seguidos de un $21.7 \%$ para las edades que se encuentran entre los 32 y 37 años de edad.

Tabla 2. Distribución según edad de tutores y tutoras del Prog
\begin{tabular}{l|ll}
\hline \multicolumn{3}{|c}{ Estudios Generales } \\
\hline Edad & Absoluto & Relativo \% \\
\hline $26-31$ años & 2 & $\mathbf{8 . 7}$ \\
$32-37$ años & 5 & $\mathbf{2 1 . 7}$ \\
$38-42$ años & 4 & $\mathbf{1 7 . 4}$ \\
$43-50$ años & 6 & $\mathbf{2 6 . 1}$ \\
51 años o más & 6 & $\mathbf{2 6 . 1}$ \\
\hline Total & $\mathbf{2 3}$ & $\mathbf{1 0 0 . 0}$
\end{tabular}

Fuente: Elaboración propia

En la tabla 3 se evidencia, respecto a la atención de estudiantes con discapacidad, que un $91.8 \%$ de las personas entrevistadas están totalmente de acuerdo o de acuerdo en que las experiencias previas del profesor-tutor y de la profesora-tutora son importantes para el desarrollo de las estrategias que utilizan con esta población. Este porcentaje tan alto se podría interpretar como consecuencia de las funciones del profesor-tutor que propone García Arieto (2001), entre las cuales destacan estar capacitado para utilizar correctamente las estrategias metodológicas más adecuadas para cada situación de aprendizaje. 
Tabla 3. Importancia que las personas entrevistadas le otorgan a las experiencias del tutor o de la tutora para el desarrollo de estrategias que buscan mejorar la atención de estudiantes con alguna discapacidad en la UNED

\begin{tabular}{l|ll}
\hline Importancia de las experiencias & Absoluto & Relativo \% \\
\hline Totalmente de acuerdo & 17 & $\mathbf{7 3 . 9}$ \\
De acuerdo & 4 & $\mathbf{1 7 . 4}$ \\
No responde & 1 & 4.3 \\
No aplica & 1 & 4.3 \\
\hline Total & $\mathbf{2 3}$ & $\mathbf{1 0 0 . 0}$ \\
\hline
\end{tabular}

Fuente: Elaboración propia

Con respecto al trato que los y las estudiantes con discapacidad reciben de los profesores-tutores y las profesoras-tutoras, un $43.5 \%$ de las personas entrevistadas se encuentra bastante de acuerdo o podría estar de acuerdo con que la atención brindada por el profesor-tutor se caracteriza por la indiferencia, mientras que un $39.1 \%$ no está de acuerdo.

Figura 2. Opinión sobre la atención que los y las estudiantes con discapacidad reciben de los tutores y las tutoras (I cuatrimestre 2011)

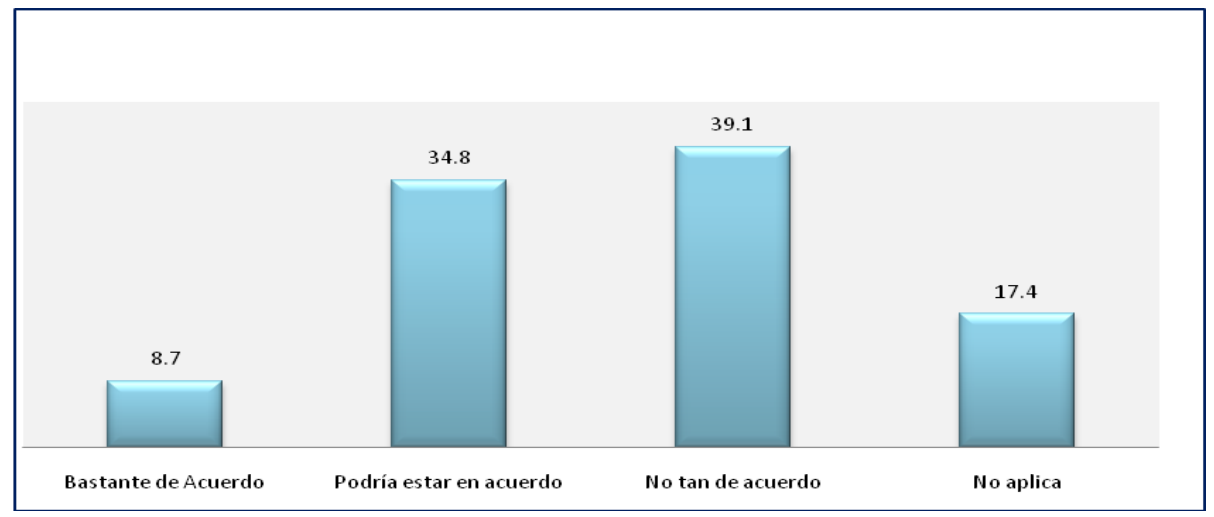

Fuente: Elaboración propia

En la tabla 4, respecto a la atención que los entrevistados y las entrevistadas dicen brindarle a la población estudiantil con alguna discapacidad, un 69.5\% menciona que siempre o muchas veces realiza alguna adecuación del entorno 
para ofrecerle mayor comodidad al o a la estudiante. Mientras que un $26.1 \%$ menciona que pocas veces o alguna vez realiza alguna adecuación del entorno. Las respuestas de las personas entrevistadas sobre la adecuación del entorno coinciden con los planteamientos de Arnáiz (2000), que manifestó que «educar en la diversidad significa ejercer los principios de igualdad y equidad a los que todo ser humano tiene derecho, lo que conlleva desarrollar unas estrategias de enseñanza-aprendizaje que personalicen la enseñanza en un marco y dinámica de trabajo para todos» (p.38).

Además, es importante destacar que para considerar una atención de calidad esta debería ser dinámica, es decir, cambiar según el contexto y las directrices institucionales.

\section{Tabla 4. Frecuencia con la que el tutor o la tutora dice adecuar el entorno para la atención de estudiantes con alguna discapacidad en busca de su comodidad}

\begin{tabular}{lll}
\hline Adecuación del entorno & Absoluto & Relativo \% \\
\hline Siempre & 13 & $\mathbf{5 6 . 5}$ \\
Muchas veces & 3 & $\mathbf{1 3 . 0}$ \\
Pocas Veces & 2 & $\mathbf{8 . 7}$ \\
Alguna Vez & 4 & $\mathbf{1 7 . 4}$ \\
No aplica & 1 & $\mathbf{4 . 3}$ \\
\hline Total & $\mathbf{2 3}$ & $\mathbf{1 0 0 . 0}$
\end{tabular}

Fuente: Elaboración propia

La tabla 5 muestra la opinión-percepción de los profesores-tutores y las profesoras-tutoras respecto al conocimiento que tienen sobre la normativa que tutela los derechos de los y las estudiantes con alguna discapacidad. El 56.5\% de las personas entrevistadas considera que siempre o muchas veces los tutores y las tutoras desconocen la normativa. 
Asimismo, la tabla 6, respecto a la información sobre la atención a personas con discapacidad, señala que un $65.2 \%$ de la población entrevistada nunca ha recibido información, mientras que un $34.7 \%$ en algún momento la recibió.

Estos altos porcentajes de desconocimiento se pueden interpretar como una desventaja para lograr una educación inclusiva de calidad, porque las investigaciones identifican como clave desarrollar estrategias de comunicación y establecer un aprendizaje dialógico entre los diferentes sujetos de la educación (Ainscow, 2005; Ainscow y Miles, 2009).

Tabla 5. Percepción acerca del desconocimiento de las personas entrevistadas sobre la normativa que tutela los derechos de los y las estudiantes con alguna discapacidad

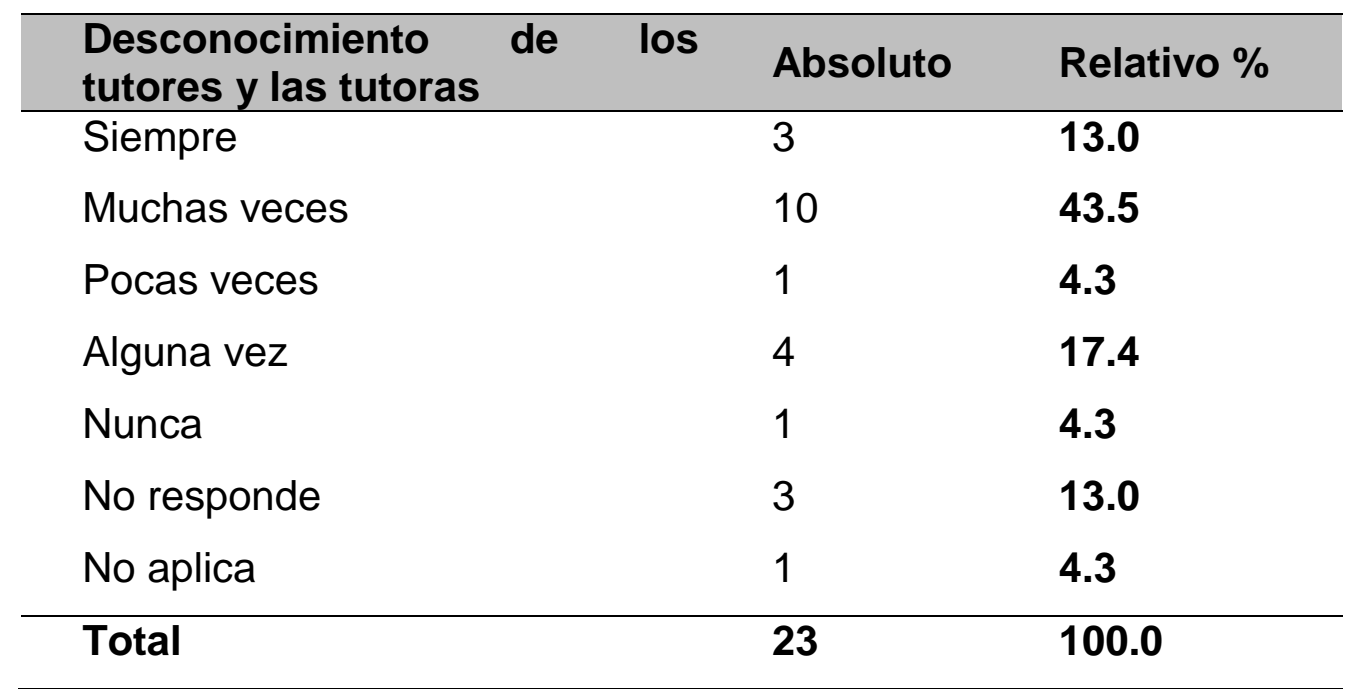

Fuente: Elaboración propia 
Tabla 6. Frecuencia con la que el profesor-tutor o la profesora-tutora recibe información sobre la atención a las personas con alguna discapacidad

\begin{tabular}{lll}
\hline $\begin{array}{c}\text { Información brindada por la } \\
\text { universidad sobre la atención a los y } \\
\text { las estudiantes }\end{array}$ & Absoluto & Relativo \% \\
\hline Pocas veces & 5 & 21.7 \\
Alguna vez & 3 & 13.0 \\
Nunca & 15 & 65.2 \\
\hline Total & $\mathbf{2 3}$ & $\mathbf{1 0 0 . 0}$ \\
\hline
\end{tabular}

Fuente: Elaboración propia

También, con respecto a la inducción para la atención de estudiantes con alguna discapacidad, un $65.2 \%$ de los entrevistados y las entrevistadas refiere que nunca ha recibido inducción alguna. Mientras que un 31.3\% menciona que en algún momento la recibió.

Con respecto a su propio desempeño, un $95.6 \%$ de las personas entrevistadas señala que siempre o muchas veces se han sentido satisfechas.

Tabla 7. Percepción satisfactoria del propio desempeño como tutor o tutora en la atención a estudiantes con alguna discapacidad

\begin{tabular}{lll}
\hline Desempeño satisfactorio & Absoluto & Relativo \% \\
\hline Siempre & 11 & 47.8 \\
Muchas veces & 11 & 47.8 \\
No aplica & 1 & 4.3 \\
\hline Total & $\mathbf{2 3}$ & $\mathbf{1 0 0 . 0}$ \\
\hline
\end{tabular}

Fuente: Elaboración propia 
Un $78.3 \%$ de las personas entrevistadas considera que la coordinación entre las diferentes unidades administrativas y académicas es insuficiente para la correcta aplicación de los derechos que protegen a las personas con alguna discapacidad.

Figura 3. Percepción de insuficiencia en la coordinación entre unidades académicas y administrativas responsables de velar por la aplicación de los derechos que protegen a las personas con discapacidad (I cuatrimestre)

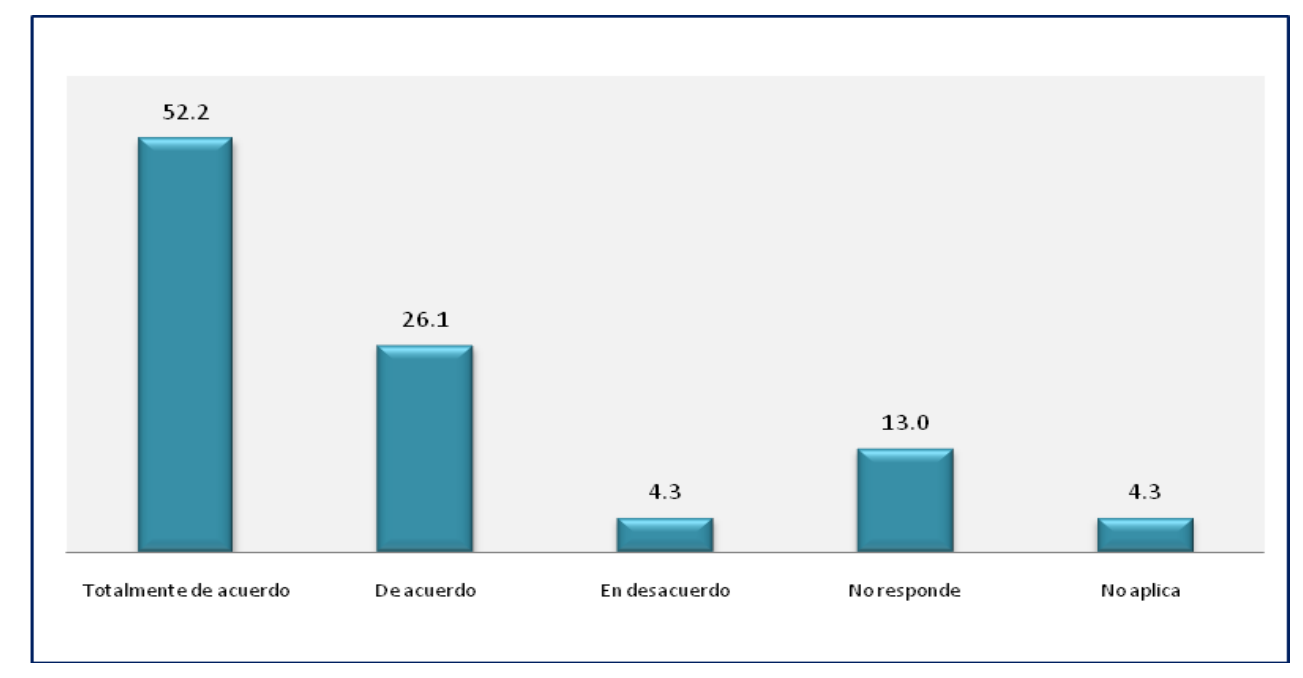

Fuente: Elaboración propia

Con respecto a la experiencia de brindar tutorías presenciales a estudiantes con discapacidad, un $56.5 \%$ de las personas entrevistadas menciona haber experimentado en algún momento tensión. Mientras que un 39.1\% expresa nunca haber experimentado tensión bajo estas circunstancias. Con relación a esta percepción, en los textos de atención a la diversidad se apunta hacia el perfeccionamiento profesional del profesorado universitario, específicamente en cuanto a la preparación del personal docente en prácticas más inclusivas (Ruiz, Mas, Tejada y Navío, 2008). 
Tabla 8. Vivencia de tensión en las personas entrevistadas cuando han tenido que trabajar las tutorías presenciales con estudiantes con alguna discapacidad

\begin{tabular}{lll} 
Vivencia de tensión & Absoluto & Relativo \% \\
\hline Siempre & 3 & 13.0 \\
Muchas veces & 2 & 8.7 \\
Pocas veces & 2 & 8.7 \\
Alguna vez & 6 & 26.1 \\
Nunca & 9 & 39.1 \\
No aplica & 1 & 4.3 \\
\hline Total & $\mathbf{2 3}$ & $\mathbf{1 0 0 . 0}$ \\
\hline
\end{tabular}

Fuente: Elaboración propia

Asimismo, respecto a si los y las estudiantes con alguna discapacidad cuentan con la atención de personas docentes especializadas, la tabla 9 muestra que un $60.8 \%$ está de acuerdo o podría estarlo, mientras que un $39.1 \%$ no lo está.

Tabla 9. Opinión de los profesores-tutores y las profesoras-tutoras sobre la pertinencia de que personas docentes especializadas atiendan a los y las estudiantes con alguna discapacidad

\begin{tabular}{lll}
\hline Opinión & Absoluto & Relativo \% \\
\hline Totalmente de acuerdo & 7 & 30.4 \\
De acuerdo & 4 & 17.4 \\
Podría estar de acuerdo & 3 & 13.0 \\
No tan de acuerdo & 8 & 34.8 \\
Totalmente en desacuerdo & 1 & 4.3 \\
\hline Total & 23 & 100.0
\end{tabular}

Fuente: Elaboración propia 
Un $73.9 \%$ de las personas entrevistadas no percibe ninguna dificultad en el desarrollo de sus tutorías cuando hay estudiantes con alguna discapacidad. Mientras que un $26 \%$ señala que, ante la presencia de estos y estas estudiantes, brindar las tutorías podría ser más difícil.

Figura 4. Percepción de la dificultad en el desarrollo de la tutoría cuando hay estudiantes con alguna discapacidad (I cuatrimestre 2011)

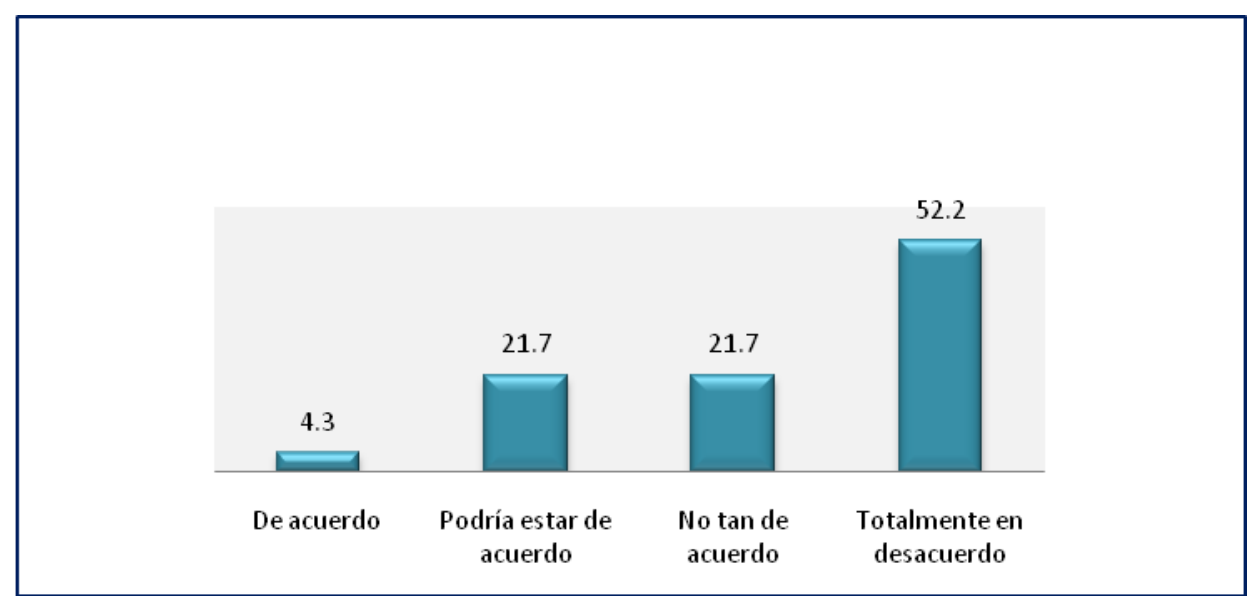

Fuente: Elaboración propia

En cuanto al protocolo para la atención de estudiantes con alguna discapacidad, un $52.2 \%$ de los entrevistados y las entrevistadas no lo conocen, solo un $17.3 \%$ afirma conocerlo y un $21.7 \%$ no sabe que responder al respecto. 
Figura 5. Conocimiento de los profesores y las profesoras de la existencia de un protocolo para la atención de estudiantes con alguna discapacidad (I cuatrimestre 2011)

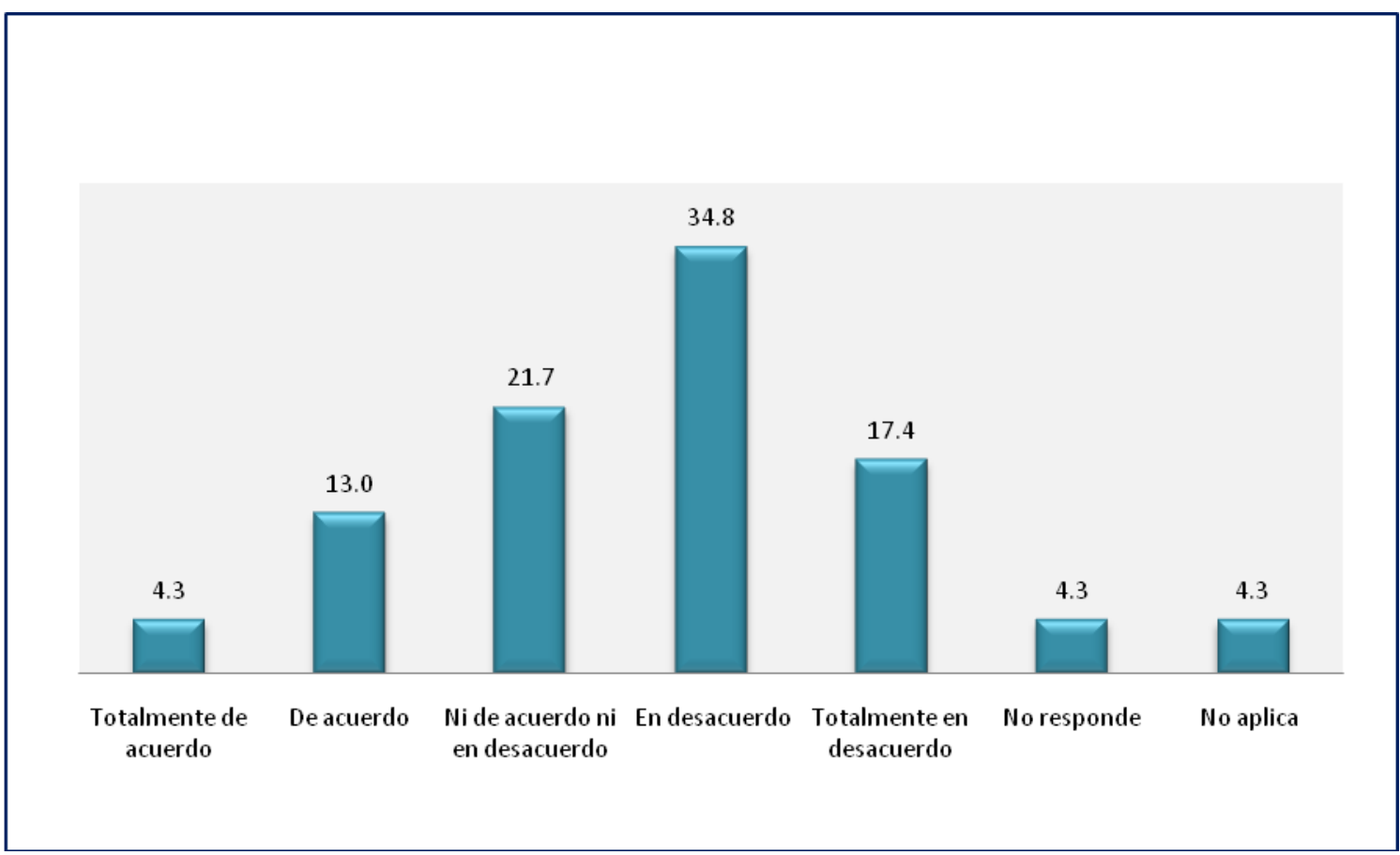

Fuente: Elaboración propia

De manera que, para considerar una gestión de calidad, la institución debe aportarle al profesorado los apoyos normalizados. Tal como lo expresaron Sarramona, López y Rodríguez (2010), la administración debe estar abierta al diálogo con todos los sectores de la comunidad educativa para garantizar el conocimiento de sus necesidades y la implicación de esos sectores en la consecución de las metas educativas.

\section{Conclusiones}

Es posible concluir que en Costa Rica la educación inclusiva tiene una legislación que garantiza políticas más específicas para la población con discapacidad, además, que en la educación superior en general y en la UNED en particular existe un esfuerzo concreto para la aplicación de la legislación. 
También, desde los postulados de misión y visión, se puede afirmar que la UNED ha logrado crear una cultura inclusiva, la cual queda plasmada en diferentes documentos institucionales donde se hacen explícitos sus valores sobre la inclusión. No obstante, desde la óptica de la aplicación práctica de estos procesos, es necesario contar con instrumentos para su evaluación y así lograr el aseguramiento de la calidad.

La búsqueda de una gestión con calidad trae consigo un incremento en las exigencias para el personal académico, como la especialización en la atención a estudiantes con discapacidad, debido a que esta labor tiene un importante grado de preparación previa. Por lo que se puede considerar como primordial la capacitación de profesores-tutores y profesoras-tutoras que trabajen con esta población, con el objetivo de contar con un cuerpo docente capacitado y sensibilizado.

Asimismo, se puede afirmar que la institución no está desarrollando las acciones pertinentes para que los y las docentes estén en capacidad de atender a esta población. Para que la atención se considere de calidad debe incluir no solo la accesibilidad física, sino también de conocimiento, por lo que debería estudiar sus currículos a la luz de la discapacidad que presente el o la estudiante, y, en caso de ser necesario, coordinar su atención con diversas instancias de la UNED.

Es posible aseverar que el personal académico del Programa de Estudios Generales asocia la atención que reciben los y las estudiantes con discapacidad con una carencia de información y sensibilización en cuanto a la real importancia de desarrollar una gestión académica con calidad.

Un indicador de calidad para la institución es contar con un protocolo para la atención de estudiantes con necesidades especiales (UNED 2009), no obstante, 
un porcentaje alto de docentes manifestó no conocerlo, por lo tanto, sería oportuno realizar un esfuerzo de socialización del mismo.

Finalmente, la investigación presenta limitaciones que abren opciones de indagación, como el tema de la adecuación de los currículos —que incluyan las adaptaciones de los materiales, los contenidos y las evaluaciones de acuerdo con las cuatro discapacidades básicas (discapacidad física, problemas visuales, sordera, discapacidades múltiples) - y el seguimiento a graduados y graduadas de la población con discapacidad.

\section{Referencias}

Asamblea Legislativa de la República de Costa Rica (1996). Ley N. 7600 Igualdad de oportunidades para las personas con discapacidad. San José, Costa Rica.

Arnáiz, P. (2000). Educar en y para la diversidad. En F. J., Soto y J. A., López, Nuevas tecnologías, viejas esperanzas: las nuevas tecnologías en el ámbito de la discapacidad y las necesidades especiales (p.29-40.). Murcia, España: Consejería de Educación y Cultura.

Ainscow, M. (2005). La mejora de la escuela inclusiva. Cuadernos de Pedagogía, 349, 78-83.

Ainscow, M. y Miles, S. (2009). Desarrollando sistemas de educación inclusiva. ¿Cómo podemos hacer progresar las políticas? En C., Giné (coord.), La educación inclusiva. De la exclusión a la plena participación de todo el alumnado (pp. 161-170). Barcelona, España: Horsor.

Cano, E. (1999). Evaluación de la Calidad Educativa. Madrid, España: Editorial la Muralla.

Creswell, J. W. (2003). Research Design: Qualitative, Quantitative and Mixed Methods Approaches (2. ${ }^{a}$ ed.) University of Nebraska, Lincoln: SAGE Publications.

García, L. (2001). La educación a distancia. De la teoría a la práctica. Barcelona, España: Ariel. 
Giraldo, U., Abad, D. y Díaz, E. (2005). Bases para una política de calidad de la educción superior en Colombia. Recuperado de http://www.ascolfa.edu.co

Hernández, R., Fernández, C. y Baptista, P. (2010). Metodología de la Investigación (5. ${ }^{\mathrm{a}}$ ed.). México, DF: MacGraw-Hill Interamericana Editores.

Instituto Interamericano de Derechos Humanos (2007). Derecho de las personas con discapacidad (módulo 6). San José, Costa Rica.

Juran, J. M. (1996). Juran y la calidad por el diseño. Madrid, España: Ediciones Díaz de Santos.

ONU (2007). Convención sobre los derechos de las personas con discapacidad. Resolución aprobada por la Asamblea General número 61/106. Recuperado de http://www.un.org/spanish/disabilities/default.asp?id=617

UNED (2009) Protocolo para Atención de las Personas con Discapacidad. Vicerrectoría Ejecutiva, San José, Costa Rica.

Ruiz, C., Mas, O., Tejada, J. y Navío, A. (2008). Funciones y escenarios de actuación del profesor universitario. Apuntes para la definición de un perfil basado en competencias. Revista de Educación Superior, XXXVII 2(146), 115-132.

Sarramona, I., López, J. y Rodríguez, T. (2010). Participación y calidad de la educación. Aula Abierta, 38(1), 3-14.

Universidad Estatal a Distancia (2008). Plan académico 2008-2011. Vicerrectoría Académica, San José, Costa Rica.

UNESCO (junio, 2009). Conferencia Mundial sobre la Educación Superior 2009. La nueva dinámica de la educación superior y la investigación para el cambio social $y$ el desarrollo. Recuperado de http://www.google.com/url?url=http://www.iesalc.unesco.org.ve/dmdocumen ts/comunicado_cmes09es.pdf\&rct=j\&sa=U\&ei=S9OETrvzN4jagAfdcUl\&ved=0CBMQFjAB\&sig2=jHSORerum_L4ktaaDrPF7w\&q=conferencia+ mudial+educacion+superior+2009\&usg=AFQjCNGGM2uhUGjibsMA4ZvqyX _2s_u4yw 